\title{
Accuracy of computer-assisted mandibular reconstructions with free fibula flap: Results of a single-center series
}

\author{
Femke Goormans $^{\mathrm{a}}$, Yi Sun ${ }^{\mathrm{a}, \mathrm{b}, *}$, Michel Bila ${ }^{\mathrm{a}, \mathrm{b}}$, Joseph Schoenaers ${ }^{\mathrm{a}}$, Joris Geusens ${ }^{\mathrm{a}}$, \\ Heinz-Theo Lübbers ${ }^{c}$, Wim Coucke ${ }^{\mathrm{d}}$, Constantinus Politis ${ }^{\mathrm{a}, \mathrm{b}}$ \\ ${ }^{a}$ Department of Oral and Maxillofacial Surgery/Faculty of Medicine KU Leuven, University Hospitals Leuven, Campus Sint-Rafaël, Kapucijnenvoer 33, 3000 Leuven, \\ Belgium \\ ${ }^{\mathrm{b}}$ Department of Imaging \& Pathology, OMFS IMPATH Research Group, Kapucijnenvoer 33, 3000 Leuven, Belgium \\ ${ }^{\mathrm{c}}$ Clinic for Cranio-Maxillofacial Surgery, University Hospital of Zurich, Frauenklinikstrasse 24, CH-8091 Zurich, Switzerland \\ ${ }^{\mathrm{d}}$ Sciensano, J. Wytsmanstraat 14, 1050 Brussels, Belgium
}

\section{A R T I C L E I N F O}

\section{Keywords:}

Mandibular reconstruction

Surgery

Computer-aided design

Computer-aided manufacturing

Data accuracy

Printing, three-dimensional

Imaging, three-dimensional

Fibula

Head and neck neoplasms

Surgical fixation devices

\begin{abstract}
A B S T R A C T
Objectives: We evaluated the accuracy of computer-assisted mandibular reconstructions.

Patients and methods: We retrospectively reviewed data for 26 patients who had mandibular reconstruction with a microvascular free fibula flap, January 2015 to June 2018. Postoperative mandible models were obtained from computed tomography scans. After registering the models to the corresponding preoperative plan, we performed comparative measurements. Patients were grouped by condylar involvement and subdivided based on number of fibular segments used for reconstruction. For each segment, we measured length and osteotomy angles. For the final postoperative outcome, we compared intercoronoid, intergonial, and anteroposterior distances and intersegmental plane shift.

Results: Means (SD) for deviation of each osteotomy angle and fibular segment length were $1.98^{\circ}(2.98)$ and $1.78 \mathrm{~mm}$ (2.69), respectively, remaining constant across subgroups. Other mean values were as follows: intercoronoid distance deviation, $3.86 \mathrm{~mm}$ (range, $0.20-11.21 \mathrm{~mm}$ ); intergonial distance deviation, $3.14 \mathrm{~mm}$ (range, 0.05-8.28 mm); anteroposterior distance deviation, $2.92 \mathrm{~mm}$ (range, 0.03-8.49 $\mathrm{mm}$ ); and intersegmental plane shift, $11.00^{\circ}$ (range, $2.76-24.15^{\circ}$ ). Where the condyle was preserved, the intercoronoid and intergonial deviation means differed significantly (respectively $5.02 \mathrm{~mm}$ and $4.88 \mathrm{~mm}$, both $P<0.05$ ) for one-segmented and threesegmented fibular reconstructions. Furthermore, reconstructions involving the condylar region compared with condyle preservation showed significantly different intersegmental plane shifts $\left(7.18^{\circ} ; P<0.05\right)$.

Conclusion: Computer-assisted surgery provides cutting guides for obtaining accurate fibular segments, but current fixation methods lead to inaccuracies and reproducibility errors. In multisegmental transfer with condylar involvement, computer-assisted fixation is recommended to ensure accuracy of the preoperative plan.
\end{abstract}

\section{Introduction}

Functional reconstruction of oromandibular defects has long been a surgical challenge [1]. The causes of deformities in this region vary from oncologic pathology to iatrogenic conditions such as osteoradionecrosis (ORN) [2]. Loss of mandibular continuity causes aesthetic and functional deficits with resulting impairments in swallowing, speech, and mastication.

Microvascular free fibula transfer is currently considered the gold standard for reconstructing large mandibular defects $[3,4]$. In recent years, computer-aided design (CAD)/computer-aided manufacturing (CAM) techniques have led to better outcomes in terms of morphology and function $[5,6]$. Preoperative planning is used to identify resection margins and visualize reconstruction options. The cutting guides used for mandibular and fibular osteotomies ensure accurate execution of the preoperative plan, minimizing the time required for the fibular osteotomy and eliminating the need for intraoperative freehand contouring or measurement. These improvements decrease handling trauma to fibular segments and allow for precise positioning with optimal bony approximation [6]. Acceleration of this previously time-

\footnotetext{
Abbreviations: CAD, computer-assisted design; CAM, computer-assisted manufacturing; SL, segment length; OA, osteotomy angle

* Corresponding author at: Department of Oral and Maxillofacial Surgery, University Hospitals Leuven, Campus Sint Rafaël, Kapucijnenvoer 33, 3000 Leuven, Belgium.

E-mail address: yi.sun@uzleuven.be (Y. Sun).
} 
consuming step decreases surgical time and duration of ischemia [7]. Kääriäinen et al., for example, reported a mean ischemia time of $99 \mathrm{~min}$ with CAD/CAM, compared to 120-180 min with conventional techniques [8]. However, this method has several drawbacks, including increased preoperative planning time and difficulty implementing intraoperative adaptation to changes in resection or reconstruction $[9,10]$.

Previous studies comparing CAD/CAM to conventional methods have indicated significant improvement of technical accuracy with digital guidance [11], but final outcomes often still contain deviances of several millimeters. The causes of outcome inaccuracies, however, remain unclear. We hypothesized that the fixation method is a major determinant of postoperative accuracy. To detect recurrent errors in outcome, we evaluated the surgical results of virtually planned mandibular reconstructions in 26 cases.

\section{Patients and methods}

\section{Patients}

This retrospective study included 26 patients, who all underwent mandibular reconstruction with a vascularized free fibula flap at the Department of Oral and Maxillofacial Surgery of Leuven University Hospitals. From January 2015 to June 2018, we reviewed 41 cases and excluded 15. Eleven cases were excluded for unavailable postoperative computed tomography (CT) scans: six had scans that did not meet the criterion of a minimum slice thickness of $1.0 \mathrm{~mm}$, three did not have postoperative CT performed within 6 months after surgery, and two patients died before a postoperative CT was performed. Another four cases were excluded because of intraoperative changes warranting deviation from the computer-assisted planning. An important reason for deviation from the planning was different positioning of the cutting guide on the mandible because of interference from soft tissue or because a broader resection was warranted given tumor progression. The same surgical team performed each operation at the same institution.

Patient characteristics are presented in Table 1 . The male/female distribution was $17 / 9(65 \% / 35 \%)$, and the mean age at reconstruction was 52.5 years (range, 6-77 years). Primary indications for reconstruction were malignancy $(n=18)$ and ORN $(n=8)$. Postoperative radiotherapy (RT) ( 60 or $66 \mathrm{~Gy}$, in daily fractions of $2 \mathrm{~Gy}$ ) was administered for 10 out of 26 patients. The study was approved by the research ethics committee of our university hospital (MP004576).

\section{Preoperative planning and surgical templates}

For all patients, CT scans of the craniofacial skeleton and fibula (with concomitant CT angiography) were obtained preoperatively. The scans were imported into a three-dimensional (3D) software program (Proplan, Materialise, Leuven, Belgium). The medical imaging engineer at our institution performed the virtual planning. Upon determination of appropriate margins, a virtual resection was completed, followed by evaluation of fibular segment lengths (SLs) and number of osteotomies. Based on these data, 3-matic software (Materialise, Leuven, Belgium) was used to design a reconstructed mandibular model, fixation tray, and cutting guides for mandibular and fibular osteotomies, which were printed using a Connex 350 3D printer (Stratasys, Eden Prairie, MN, USA) (Fig. 1). For each patient, the reconstruction plate was manually bent based on the 3D-printed reconstructed mandible model, which included the reconstruction with one or more fibular segments. Surgeon preference determined the type of fixation plate used for the reconstruction. We used $2.3 \mathrm{~mm}(\mathrm{n}=11)$ and $2.7 \mathrm{~mm}(\mathrm{n}=15)$ locking reconstruction plates with monocortical locking screws (KLS Martin Group, Tuttlingen, Germany). A fixation tray was used for guided placement of the reconstruction plate.
Table 1

Patient characteristics.

\begin{tabular}{|c|c|c|c|c|c|c|}
\hline $\mathrm{N}$ & Sex & Age (y) & Indication & $\begin{array}{l}\text { Condyle } \\
\text { preserved }\end{array}$ & $\begin{array}{l}\text { Number of fibular } \\
\text { segments }\end{array}$ & Group \\
\hline 1 & $\mathrm{~F}$ & 54 & SCC & Yes & 1 & A1 \\
\hline 2 & M & 55 & SCC & Yes & 1 & A1 \\
\hline 3 & M & 69 & ORN & Yes & 1 & A1 \\
\hline 4 & M & 69 & SCC & Yes & 1 & A1 \\
\hline 5 & $\mathrm{~F}$ & 52 & ORN & Yes & 2 & A2 \\
\hline 6 & M & 63 & SCC & Yes & 2 & A2 \\
\hline 7 & M & 51 & SCC & Yes & 2 & A2 \\
\hline 8 & M & 63 & SCC & Yes & 2 & A2 \\
\hline 9 & $\mathrm{~F}$ & 74 & SCC & Yes & 2 & A2 \\
\hline 10 & M & 59 & ORN & Yes & 2 & A2 \\
\hline 11 & M & 69 & SCC & Yes & 2 & A2 \\
\hline 12 & M & 77 & SCC & Yes & 3 & A3 \\
\hline 13 & M & 61 & SCC & Yes & 3 & A3 \\
\hline 14 & $\mathrm{~F}$ & 56 & SCC & Yes & 3 & A3 \\
\hline 15 & $\mathrm{~F}$ & 63 & ORN & Yes & 3 & A3 \\
\hline 16 & $\mathrm{~F}$ & 66 & SCC & Yes & 3 & A3 \\
\hline 17 & M & 51 & ORN & Yes & 3 & A3 \\
\hline 18 & M & 47 & ORN & Yes & 3 & A3 \\
\hline 19 & $\mathrm{~F}$ & 54 & SCC & No & 1 & B1 \\
\hline 20 & M & 55 & ORN & No & 1 & B1 \\
\hline 21 & $\mathrm{~F}$ & 21 & RMS & No & 1 & B1 \\
\hline 22 & M & 13 & OS & No & 1 & B1 \\
\hline 23 & M & 47 & SCC & No & 2 & B2 \\
\hline 24 & M & 14 & RMS & No & 2 & B2 \\
\hline 25 & $\mathrm{~F}$ & 6 & ES & No & 2 & B2 \\
\hline 26 & M & 56 & ORN & No & 2 & B2 \\
\hline
\end{tabular}

Abbreviations: F: female; M: male; SCC: squamous cell carcinoma; ORN: osteoradionecrosis; RMS: rhabdomyosarcoma; ES: Ewing sarcoma; OS: osteosarcoma; A: condyle preserved; B: condyle involved in resection; 1-3, number of fibular segments used for reconstruction.

\section{Postoperative accuracy study}

A postoperative CT scan for assessment of bony consolidation was performed on average 3 months after treatment (range, 4 days to 187 days postoperatively; average, 82 days postoperatively). Earlier CT scans were performed for patients requiring postoperative RT or when early complications developed. Postoperative RT could not have influenced our results because accuracy measurements were performed on CT images that predated RT administration. Bony consolidation could have influenced measurements of the fibular segment accuracy, so we excluded cases where CT scans were obtained more than 6 months after surgery. An important reason for delayed CT scans was poor patient adherence to clinical follow-up.

The CT data were registered to the preoperative planning in Amira software (Thermo Fisher Scientific, Waltham, MA, USA), and the postoperative $3 \mathrm{D}$ mandible model was created. The same person performed all measurements in 3-matic software.

Through comparative measurements on the preoperative planning results and the postoperative mandible model, we determined how much postoperative outcomes deviated from the corresponding preoperative plans. We defined deviations for each individual bone segment using two indicators: the length deviation of each fibular segment (Fig. 2) and the angular deviation of each fibular osteotomy (Fig. 3A and $\mathrm{B})$. The length deviation was determined by the outer upper margin, outer lower margin, and inner median margin deviations. The angular deviation was determined by measuring the angle between each plane on the lateral and outer sides of each fibular segment.

In addition, we used four indicators to evaluated deviations between the planned and actual postoperative position of fibular segments: intersegmental angle shift, intercoronoid distance, intergonial distance, and anteroposterior distance. The intersegmental angle shift was the difference in the angles formed between the cutting plane on the 


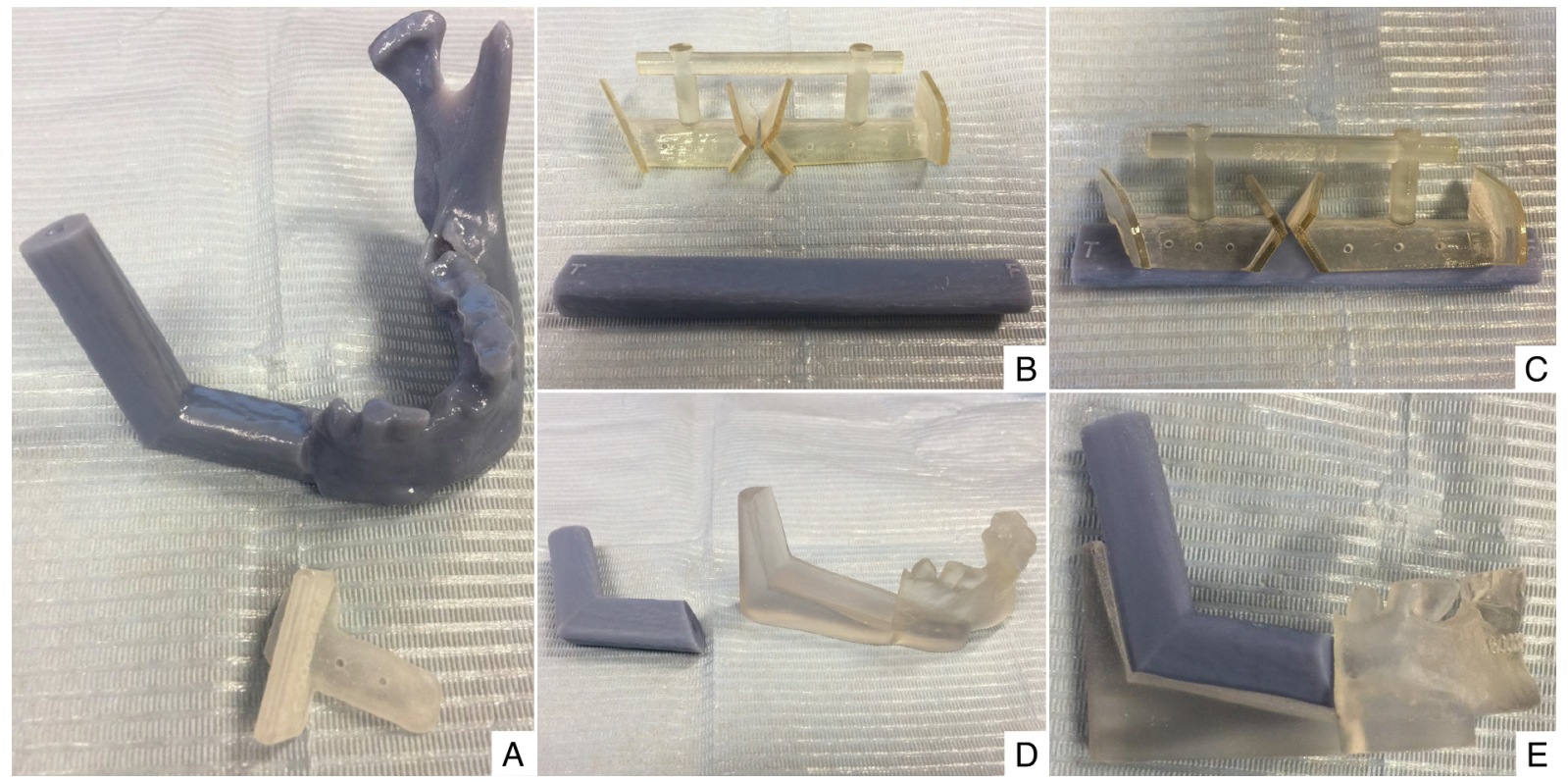

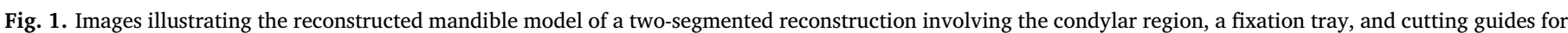

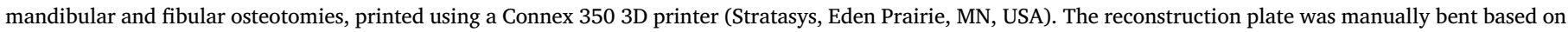

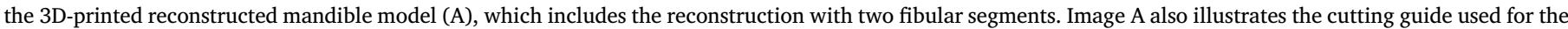

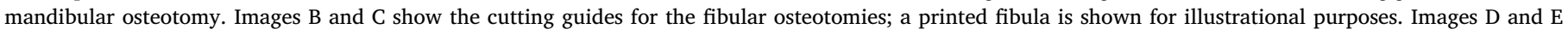
depict a fixation tray, which was used for guided placement of the reconstruction plate. Two printed fibular segments are shown for illustrational purposes.

planning and postoperative mandible models (Fig. 3C). Furthermore, we determined and compared intercoronoid distance (Fig. 4A), intergonial distance (Fig. 4B), and anteroposterior distance (using a perpendicular line drawn from the mandibular midpoint to a plane determined by both condyles and the mandibular gonion) (Fig. 4C).

\section{Data analysis}

We created two comparison groups from the obtained data, one group in which the condyle was preserved and the other in which it was involved in the resection. Each of these groups was split into three subgroups of one-segmented, two-segmented, or three-segmented fibular transfer. We compared the results among subgroups and evaluated the impact of condylar involvement on reconstruction accuracy.

\section{Statistics}

A certified statistician performed the analyses of differences between preoperative planning measurements and postoperative outcome. Means and standard deviations were calculated for each parameter. Patients were subsequently subdivided into comparison groups. For analyzing segment length (SL) and osteotomy angle (OA), we used a linear mixed model with the patient as a random factor and group as a fixed factor. We applied analysis of variance to the other parameters, with the group as a fixed factor, and tested residual values for normality and homoscedasticity. Statistical significance was estimated at $P<0.05$ for each statistical test.

\section{Results}

\section{Postoperative course and complications}

All ischemia times were $<4.00 \mathrm{~h}$, with most ischemia times $<$ $2.00 \mathrm{~h}$. The minimal follow-up period was 10 months. The overall survival rate of fibular flaps included in our study was $96.15 \%$.

Complications were categorized as acute and late. Acute complications ( $<6$ weeks postoperatively) were mostly vascular problems, such as hemorrhage $(n=2)$ or venous congestion $(n=1)$, that necessitated immediate intervention. Insufficient blood supply of the skin island in three cases led to skin necrosis and surgical debridement. Late complications ( $>6$ weeks postoperatively) were chronic infection, necrosis, or trismus. Removal of the fixation plate $(n=1)$ and bony reconstruction $(n=1)$ was required for one patient each, respectively, because of chronic osteomyelitis and necrosis. Two out of ten patients who received postoperative RT developed low-grade ORN, which was treated conservatively. Trismus (defined by a mouth opening of $<30$ $\mathrm{mm}$ ) was described for three cases; however, two of these were ORN cases that had already shown trismus preoperatively.

Implant placement was performed several months postoperatively for $12(46.15 \%)$ of the included patients. None of the included patients received dental implants perioperatively. Implant placement could not have influenced our results because accuracy measurements were performed on CT images that predated implant placement.

\section{Postoperative accuracy study}

We evaluated 50 fibular segments (Table 1). Table 2 illustrates the differences for each subgroup. The mean deviation in fibular SL was $1.74 \mathrm{~mm}$ (range, $0.02-6.10 \mathrm{~mm}$ ), and the mean angular deviation of the osteotomy planes was $1.98^{\circ}$ (range, $0.04-5.86^{\circ}$ ). The results remained constant for each subgroup and were highly accurate compared to the preoperative plans. Thus, reproducibility was optimal using 3D printed cutting guides for SLs and OAs.

For other measures, the reproducibility was less optimal. Mean values for each measure were as follows: intercoronoid distance deviation, $3.86 \mathrm{~mm}$ (range, $0.20-11.21 \mathrm{~mm}$ ); intergonial distance deviation, $3.14 \mathrm{~mm}$ (range, $0.05-8.28 \mathrm{~mm}$ ); anteroposterior distance deviation, $2.92 \mathrm{~mm}$ (range, $0.03-8.49 \mathrm{~mm}$ ); and intersegmental plane shift, $11.00^{\circ}$ (range, $2.76-24.15^{\circ}$ ). These results demonstrate a high degree of variation in final postoperative accuracy.

The condyle was preserved in 18 patients. Four patients underwent one-segmented reconstruction, seven patients had two-segmented reconstruction, and seven had three-segmented reconstruction. For eight patients, the condyle was involved in the resection; four of these 


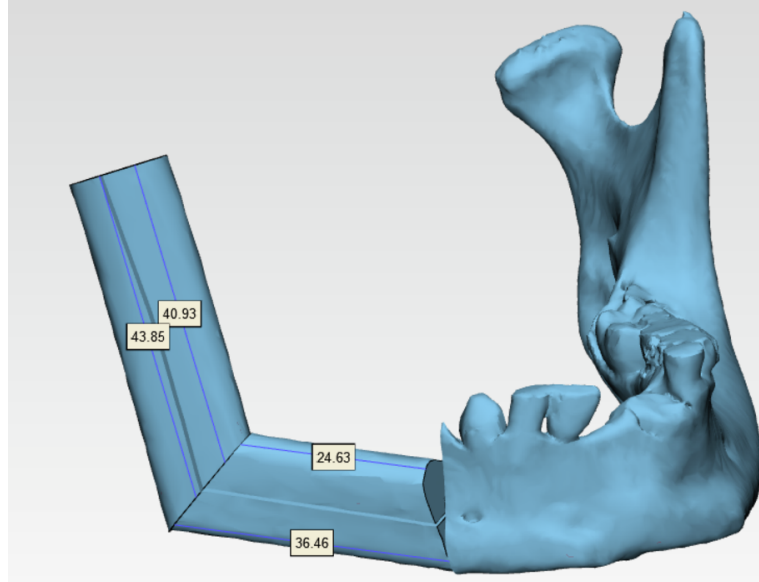

A

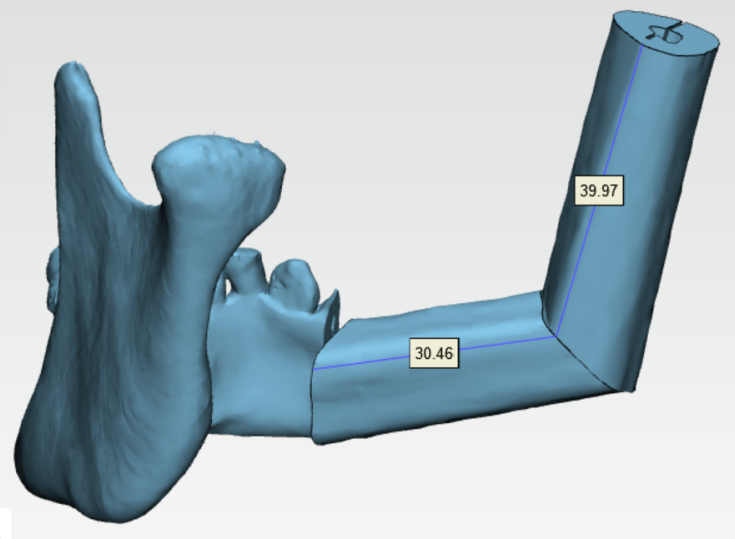

B

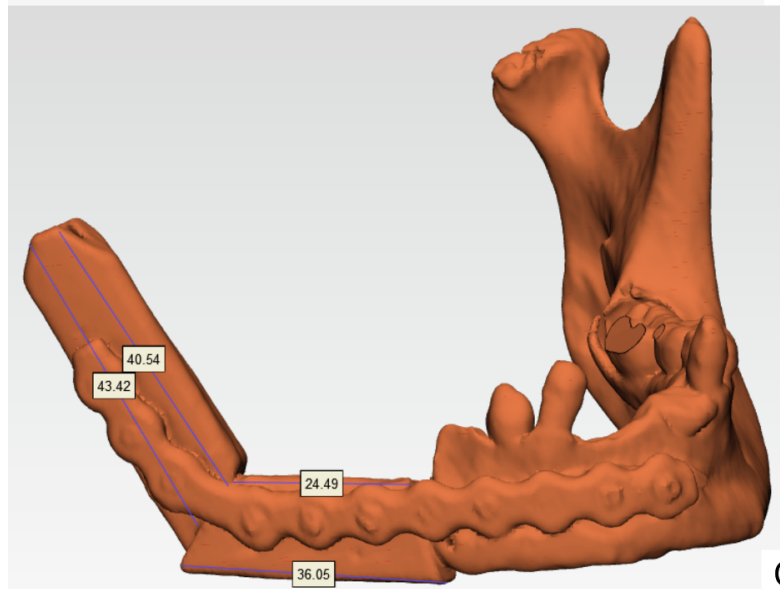

C

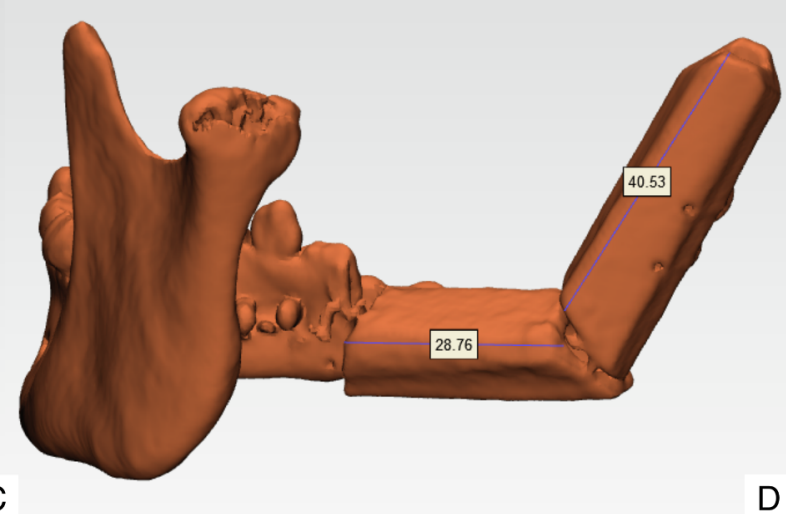

Fig. 2. Three-dimensional images illustrating the preoperative planning mandible model (blue) as well as the postoperative mandible model derived from a postoperative CT scan (orange). Images A and B show lateral and medial views, respectively, of the preoperative planning, illustrating the measurements of segment length on three different planes: the outer superior and outer inferior segment lengths (A) and the inner median segment length (B). Images C and D show lateral and medial views, respectively, of the postoperative mandible, illustrating the measurements on the corresponding planes. Evaluation of accuracy was obtained by subtracting the postoperative segment lengths from the corresponding preoperative segment lengths. (For interpretation of the references to colour in this figure legend, the reader is referred to the web version of this article.)
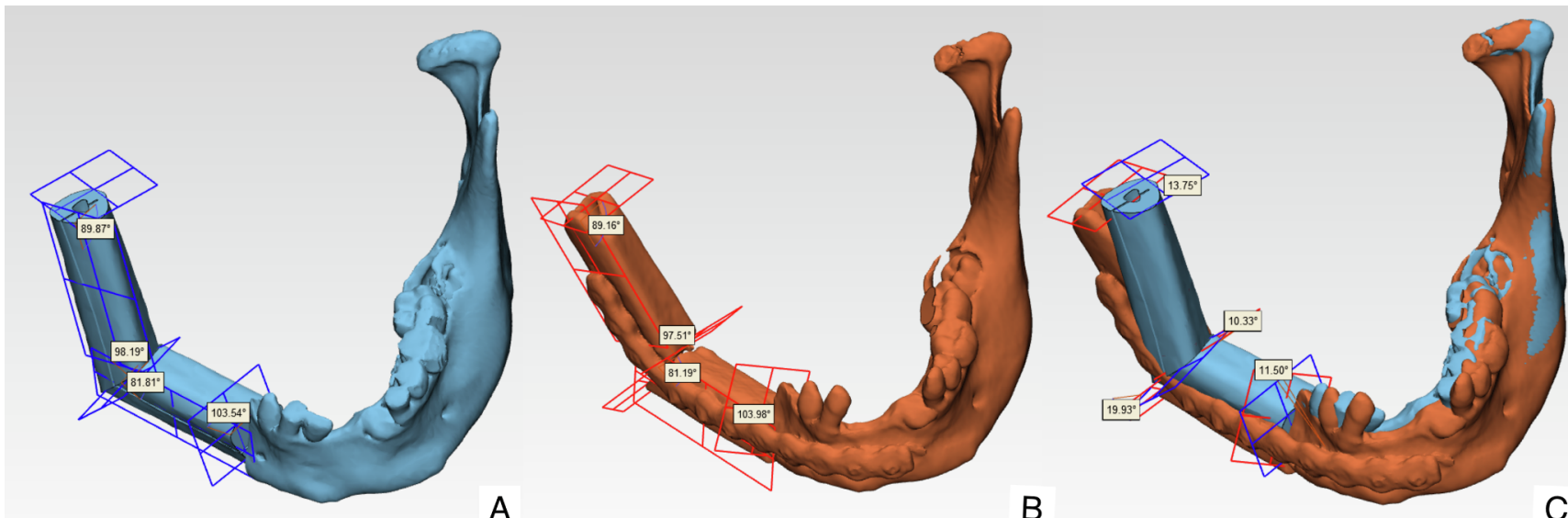

Fig. 3. Three-dimensional images show a superior view of the preoperative planning mandible model (blue) as well as the postoperative mandible model derived from a postoperative CT scan (orange). Image A illustrates the measurements of angulation on the preoperative planning, obtained by measuring the angle between each plane on the lateral and outer sides of each fibular segment. Image B illustrates the corresponding measurements of segment angulation on the postoperative mandible model. Angular deviation of osteotomy angles was obtained by subtracting the postoperative angle from the corresponding preoperative angle. Image $\mathrm{C}$ illustrates the postoperative mandible model (orange) after registration to the preoperative planning image (blue). Evaluation of intersegmental plane shift was obtained by measuring the angle between the cutting plane on the preoperative planning and the corresponding cutting plane on the postoperative mandible model. (For interpretation of the references to colour in this figure legend, the reader is referred to the web version of this article.) 


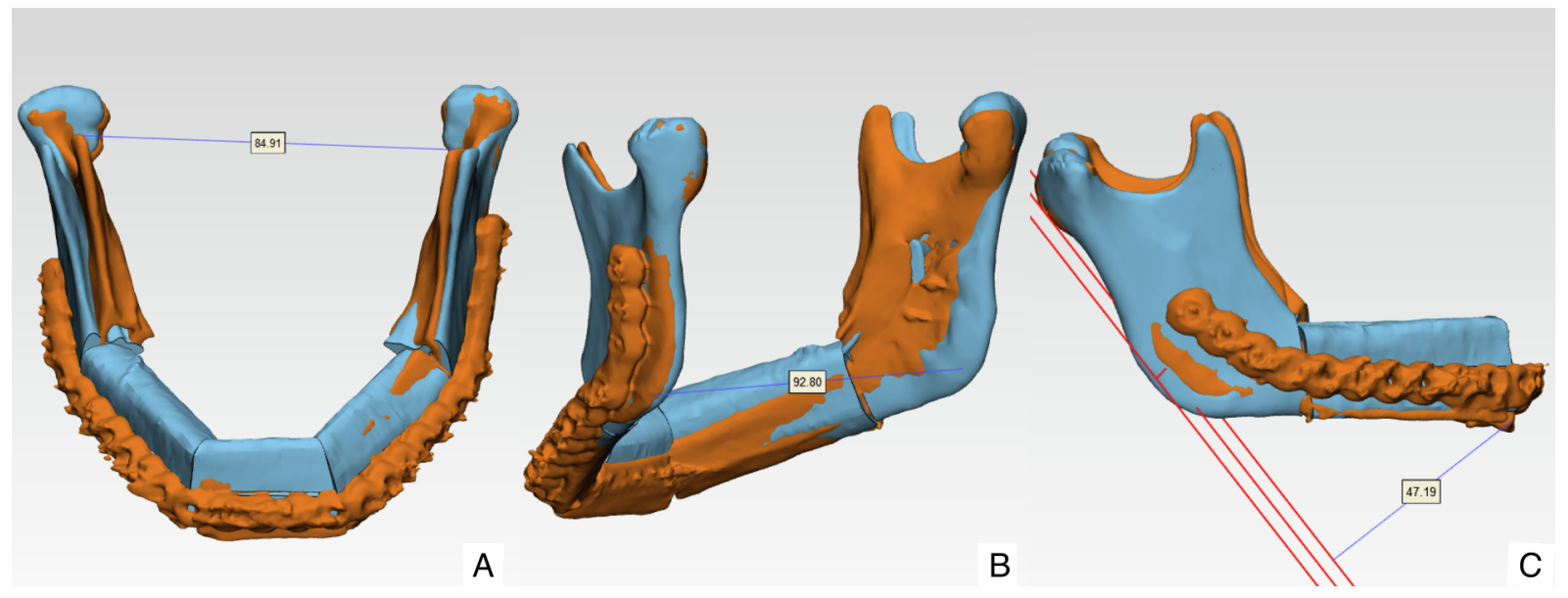

Fig. 4. Three-dimensional images illustrating the postoperative mandible model derived from a postoperative CT scan (orange) registered to the preoperative planning mandible model (blue). For illustrational purposes, only the postoperative measurement is displayed. Image A illustrates the measurement of postoperative intercoronoid distance. Image B illustrates the measurement of intergonial distance. Image C illustrates the measurement of anteroposterior distance (by drawing a perpendicular line from the mandibular midpoint to a plane determined by both condyles and the mandibular angle). (For interpretation of the references to colour in this figure legend, the reader is referred to the web version of this article.)

patients had one-segmented reconstruction, four had two-segmented reconstruction, and none had three-segmented reconstruction.

Table 3 shows results for the subgroup comparisons for individual SLs and OAs, with no significant differences. Table 4 shows results for subgroup comparison of final postoperative results. In the group with preserved condyles, mean intercoronoid and intergonial deviations differed significantly $(5.02 \mathrm{~mm}$ and $4.88 \mathrm{~mm}$, respectively; both $P<0.05$ ) for one-segmented and three-segmented fibular reconstructions, respectively. The implication of this result is that final postoperative accuracy decreases when more fibular segments are used for reconstruction.

For intersegmental plane shift, the preserved and nonpreserved condyle groups differed significantly $\left(7.18^{\circ} ; P<0.05\right)$. Thus, postoperative accuracy appears to decrease when the condyle is involved in the resection. In the nonpreserved condyle group, we could not evaluate intercoronoid deviations because in all cases, the coronoid also was resected. The intergonial deviations of one-segmented reconstructions in this group were based on only two cases because the gonion also was resected in two of four patients. Given this lower power, a finding of no significant differences was expected and confirmed.

\section{Discussion}

Practitioners are continuously challenging themselves to optimize mandibular reconstructions with the newest technologies. As such, they focus on improving outcome accuracy and predictability, reducing
Table 3

Comparison of subgroups: individual segments.

\begin{tabular}{llllll}
\hline & \multirow{2}{*}{ SL } & & & OL \\
\cline { 6 - 6 } \cline { 6 - 6 } & Difference $(\mathrm{mm})$ & $P$ & & Difference $(\mathrm{mm})$ & $P$ \\
\hline A1/A2 & 0.09 & 0.9999 & & -0.84 & 0.9157 \\
A1/A3 & 0.07 & 0.9999 & & -0.17 & 0.9998 \\
A2/A3 & 0.09 & 0.9999 & & -0.84 & 0.9157 \\
B1/B2 & 0.14 & 0.9997 & & 0.61 & 0.9808 \\
A1/B1 & -0.18 & 0.9993 & & 0.76 & 0.9707 \\
A2/B2 & -0.22 & 0.9942 & & -0.70 & 0.9254 \\
A/B & -0.20 & 0.5496 & & -0.3517 & 0.3820 \\
\hline
\end{tabular}

A: condyle preserved; B: condyle involved in resection; 1-3, number of fibular segments used for reconstruction.

Abbreviations: SL, segment length; OA, osteotomy angle.

surgical time, and optimizing postoperative function and aesthetics. To date, studies identifying causal factors in suboptimal postoperative results are lacking.

In this study, we measured several parameters to evaluate the accuracy and reproducibility of the surgical plan. Comparison of the virtual plan and the postoperative mandible model showed that the mean segment length deviation was $1.74 \mathrm{~mm}$ (range, 0.02-6.10 mm), which is similar to the deviations reported by Zhang et al. [6] and Roser et al. [12].

Table 2

Evaluation of accuracy.

\begin{tabular}{|c|c|c|c|c|c|c|c|c|}
\hline & & \multicolumn{2}{|l|}{ Individual segment } & \multicolumn{4}{|c|}{ Final postoperative result } & \multirow[b]{2}{*}{ Patients N (\%) } \\
\hline \multicolumn{2}{|c|}{ Group } & $\mathrm{SL}$ mean $(\mathrm{mm}) \pm \mathrm{SD}$ & $\mathrm{OA}$ mean $(\mathrm{mm}) \pm \mathrm{SD}$ & $\mathrm{IC}$ mean $(\mathrm{mm}) \pm \mathrm{SD}$ & IG mean $(\mathrm{mm}) \pm \mathrm{SD}$ & $\mathrm{AP}$ mean $(\mathrm{mm}) \pm \mathrm{SD}$ & IPS mean $(\mathrm{mm}) \pm \mathrm{SD}$ & \\
\hline \multirow[t]{3}{*}{$\mathbf{A}$} & 1 & $1.83 \pm 1.99$ & $1.80 \pm 0.84$ & $0.94 \pm 0.82$ & $0.29 \pm 0.33$ & $0.64 \pm 0.62$ & $4,64 \pm 2.92$ & $4(15)$ \\
\hline & 2 & $1.74 \pm 0.89$ & $2.05 \pm 1.29$ & $3.36 \pm 2.22$ & $3.93 \pm 2.61$ & $1.99 \pm 1.93$ & $8.45 \pm 3.14$ & 7 (27) \\
\hline & 3 & $1.77 \pm 1.48$ & $2.20 \pm 1.41$ & $5.96 \pm 4.57$ & $5.18^{\mathrm{a}} \pm 3.31$ & $3.86 \pm 2.33$ & $9.98 \pm 6.07$ & 7 (27) \\
\hline \multirow[t]{2}{*}{ B } & 1 & $1.65 \pm 1.03$ & $2.07 \pm 2.14$ & / & $2.70^{\mathrm{b}} \pm 2.21$ & $2.84 \pm 2.10$ & $14.22 \pm 8.50$ & $4(15)$ \\
\hline & 2 & $1.5 \pm 1.49$ & $1.51 \pm 1.11$ & / & $\mu^{\mathrm{c}}$ & $5.23 \pm 3.57$ & $16.53 \pm 5.57$ & $\begin{array}{l}4(15) \\
26(100)\end{array}$ \\
\hline
\end{tabular}

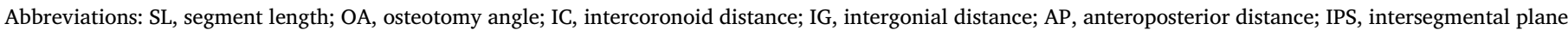
shift.

a Based on four measurements (3/7 gonion included in the resection).

b Based on two measurements (2/4 gonion included in the resection).

c No data because the gonion also was resected. 
Table 4

Comparison of subgroups: final postoperative results.

\begin{tabular}{|c|c|c|c|c|c|c|c|c|}
\hline & \multicolumn{2}{|l|}{ IC } & \multicolumn{2}{|l|}{ IG } & \multicolumn{2}{|l|}{ AP } & \multicolumn{2}{|l|}{ IPS } \\
\hline & Difference (mm) & $P$ & Difference (mm) & $P$ & Difference (mm) & $P$ & Difference (mm) & $P$ \\
\hline A1/A2 & -2.42 & 0.4583 & -3.63 & 0.1271 & -1.35 & 0.9243 & -3.81 & 0.8684 \\
\hline A1/A3 & -5.02 & 0.0360 & -4.88 & 0.0168 & -3.22 & 0.1515 & -5.33 & 0.5779 \\
\hline A2/A3 & -2.42 & 0.4583 & -3.63 & 0.1271 & -1.35 & 0.9243 & -3.81 & 0.8684 \\
\hline B1/B2 & / & / & / & / & -2.34 & 0.6627 & -2.31 & 0.9853 \\
\hline A1/B1 & / & / & 2.41 & 0.6824 & 2.20 & 0.7271 & 9.58 & 0.0841 \\
\hline A2/B2 & / & / & / & / & 3.19 & 0.1602 & 8.08 & 0.1153 \\
\hline A/B & / & / & -0.43 & 0.8548 & 1.59 & 0.1435 & 7.18 & 0.0046 \\
\hline
\end{tabular}

A: condyle preserved; B: condyle involved in resection; 1-3, number of fibular segments used for reconstruction.

Abbreviations: IC, intercoronoid distance; IG, intergonial distance; AP, anteroposterior distance; IPS, intersegmental plane shift.

Recreating the mandibular contour requires a specific OA on each side of the fibular segments. Even a small difference in this angle can account for a difference in mandibular height and in the resulting mandibular angle. Zhang et al. [6] reported angular deviation by measuring the angle formed between two fibular segments. However, this angle can be determined not only by the fibular OA but also by the positional relationship between the segments, as defined by the applied fixation method. To the best of our knowledge, the OAs of each individual fibular segment have not been reported before. Comparing outcomes to the corresponding preoperative plan, the mean angular deviation of osteotomy planes was $1.98^{\circ}$ (range, $0.04-5.86^{\circ}$ ). Our results demonstrate a minimal difference between the planned fibular OA and the resulting postoperative angle.

Deviations in SLs and OAs remained constant for each subgroup (Table 3) and were highly accurate compared to the preoperative plan. Thus, each segment was cut very precisely. Based on these data, we conclude that the cutting guides we used provided accurate OAs and directions and can be excluded as a cause of error.

The positional relationship between the segments and the mandibular remnants, however, highly influenced the postoperative result. Zhang et al. [6] evaluated the final outcome using mini-plates preoperatively bent around a printed mandible model. For intergonial deviation, they reported a mean (SD) deviation of $2.96 \mathrm{~mm}$ (1.85), which is similar to our result of $3.14 \mathrm{~mm}$ (2.95). For anteroposterior deviation, they reported a mean (SD) deviation of $4.27 \mathrm{~mm}$ (3.62), which is slightly less accurate than our result of $2.92 \mathrm{~mm}$ (2.53).

We created two comparison groups from the obtained data, a group with preserved condyle and a group in which the resection involved the condyle. These two groups were further divided based on the number of fibular segments used for reconstruction. We identified a decrease in final postoperative accuracy with increasing fibular segments used for reconstruction (Table 4). This association was even more pronounced in reconstructions involving the condylar region.

Our results illustrate the importance of using a fixation method that can maintain and guarantee the accuracy of the preoperative plan. Although we used a fixation tray for proper segment positioning, a manually bent reconstruction plate remains a cause of reproducibility error. The exact positioning for the preoperatively bent plate was not indicated, and the relation to the mandibular remnants was not specified, so residual error inhibited exact translation of the intended mandibular contour. Using a manually bent plate offers several advantages, such as faster access to planning and lower costs, but plate malpositioning can produce suboptimal mandibular contouring $[13,14]$. This problem can in turn lead to postoperative complications, such as malocclusion, facial asymmetry, limitation of mouth opening, and positional changes of the condyle $[7,13]$. Furthermore, repetitive bending of fixation plates may fatigue the metal in areas of contour, increasing the risk of premature plate fracture $[15,16]$.

To avoid these complications, surgeons attempt to improve neomandible fixation using innovative methods. In recent years, several fixation options have been described. The patient-specific 3D printed titanium plate has proven to be precise [13]. Naros et al. reported an improvement of $3.68 \mathrm{~mm}(P<0.05)$ for intergonial distance and $2.07 \mathrm{~mm}(P<0.05)$ for intercondylar angle when comparing patientspecific plates to intraoperatively bent plates. For intergonial distance, the result is considerably more accurate than our result of $3.14 \mathrm{~mm}$ using a manually bent plate. However, this method is rather expensive compared with using only printed neomandible models and surgical cutting guides, and although lead times may vary worldwide, they are generally longer $[14,17]$. This limitation may be important for malignancies that require the earliest possible treatment.

Another reliable method to further aid in accurate positioning of the reconstruction plate is the use of cutting guides with screw holes $[7,14,18]$. Fibular segments are confirmed to be in the planned position when their holes align with the screw holes in the plate, so this device ensures a correct relationship among the plate, fibular segments, and mandibular remnants [7]. Different methods have been described for obtaining screw holes in the cutting guides when using a manually bent plate, to avoid extra costs and longer lead time of patient-specific printed titanium plates. For example, Kraeima et al. [14] have presented an easy-access method, manually bending the osteosynthesis plate around a printed mandible model and subsequently drilling holes through the plate into the model. The cutting guides are then placed on the printed mandible and the screw hole is re-drilled from the printed model into the cutting guide. Bao et al. proposed another option, manually bending the fixation plate around a printed mandible model and designing the cutting guide afterwards, with the bent plate embedded [7].

Although CAD/CAM provides cutting guides for obtaining accurate fibular segments, there are no guidelines regarding the fixation method. We believe that a patient-specific 3D printed reconstruction plate would significantly improve postoperative accuracy in multisegmental reconstructions involving the condylar region.

The clinical impact of technical inaccuracies remains relatively unclear to date [11]. What is accepted as a good aesthetic outcome varies between practitioners. Of course, different anatomical areas require different levels of accuracy. For example, deviations of $1.5 \mathrm{~mm}$ are accepted for positioning of bone segments in the midface region, because a considerable amount of soft tissue covers bony inaccuracies [19]. Considering soft tissue thickness, it would be expected that mandibular reconstructions, compared to the midface, require increased accuracy to obtain an acceptable aesthetic result.

The most important goal in optimizing technical accuracy is improving postoperative function. CAD/CAM accurately represents the postoperative inter-maxillary relationship, which allows dental implant placement at the time of reconstruction in line with the antagonist dentition [11]. With this approach, the increased technical accuracy of computer-assisted surgery facilitates dental rehabilitation. In addition, 
patients who require postoperative RT have a significantly reduced risk of osteoradionecrosis with perioperative implant placement, compared with the risk associated with post-radiation implantation [20,21]. Postoperative dental rehabilitation leads to improved functional outcome and quality of life $[21,22]$. In our opinion, accuracy within a few millimeters will probably not affect postoperative function in edentulous patients; however, practitioners aim to ensure that perioperative implant placement is standard care for all patients. Of course, the patient's prognosis should be known, and preimplantation assessment of mouth opening, oral hygiene, and dental caries is required before a patient is considered for implantation [21].

Our retrospective study has several limitations. First and foremost, the comparison groups were not randomized. Second, our sample of 26 patients was relatively small, but it is important to note that this is one of the largest sample sizes of computer-assisted mandibular reconstructions with free fibula flap described to date. Third, differences in the timing of CT scans may affect accuracy measurement because shifting of bone segments can occur over time. Early shifting of the fibular segments can arise because of insufficient fixation of the mandibular segments, which is more likely in reconstructions involving the condylar region. Late shifting of segments can occur because of micromovements and bony consolidation or resorption. A segment micromovement can develop during bone remodeling, especially during RT [23]. Postoperative adhesions and scarring of surrounding soft tissue, producing traction on the fibular segments, can further influence this process. Another important long-term problem is the tendency for bone resorption. Yla-Kotola et al. [24] reported increasing reduction in fibular segment height over time, which could affect postoperative measurements. Because we included scans performed within 6 months after surgery, early bone segment shifting or bony consolidation beginning at the fibular ends could have affected our results. Finally, several extrinsic factors may affect surgical outcome, such as the heterogeneous nature of the initial pathology.

Future prospective comparative studies should compare fixation methods for each type of reconstruction and further clarify remaining errors in accuracy for each fixation method. Moreover, the relevance of accuracy in terms of function and aesthetics needs to be confirmed. CAD/CAM has evolved in a relatively short amount of time, making it probable that lead time and costs of patient-specific printed plates can be expected to decrease.

\section{Conclusion}

In the present study, we evaluated the accuracy of computer-assisted mandibular reconstructions using a manually bent plate. The findings indicate that although each individual segment is cut very precisely, the positional relationship between the segments and the mandibular remnants highly influences the final postoperative result. This effect is most important in multisegmental fibular reconstruction and when the condyle is involved in the resection. However, our results should be interpreted with caution because of the relatively small sample size. Patients should be continuously recruited to confirm the validity of these findings, and comparative studies should evaluate the optimal fixation method for each type of reconstruction.

\section{Declaration of Competing Interest}

None declared.

\section{References}

[1] Hurvitz KA, Kobayashi M, Evans GR. Current options in head and neck reconstruction. Plast Reconstr Surg 2006;118:122-33.

[2] López-Arcas JM, Arias J, Del Castillo JL, Burgueño M, Navarro I, Morán MJ, et al. The fibula osteomyocutaneous flap for mandible reconstruction: a 15-year experience. J Oral Maxillofac Surg 2010;68:2377-84.

[3] Okay D, Al Shetawi AH, Moubayed SP, Mourad M, Buchbinder D, Urken ML. Worldwide 10-year systematic review of treatment trends in fibula free flap for mandibular reconstruction. J Oral Maxillofac Surg 2016;74:2526-31.

[4] Hayden RE, Mullin DP, Patel AK. Reconstruction of the segmental mandibular defect: current state of the art. Curr Opin Otolaryngol Head Neck Surg 2012;20:231-6.

[5] Tarsitano A, Ciocca L, Scotti R, Marchetti C. Morphological results of customized microvascular mandibular reconstruction: a comparative study. J Craniomaxillofacial Surg 2016;44:697-702.

[6] Zhang L, Liu Z, Li B, Yu H, Shen SG, Wang X. Evaluation of computer-assisted mandibular reconstruction with vascularized fibular flap compared to conventional surgery. Oral Surg Oral Med Oral Pathol Oral Radiol 2016;121:139-48.

[7] Bao T, He J, Yu C, Zhao W, Lin Y, Wang H, et al. Utilization of a pre-bent platepositioning surgical guide system in precise mandibular reconstruction with a free fibula flap. Oral Oncol 2017:75:133-9.

[8] Kääriäinen M, Kuuskeri M, Gremoutis G, Kuokkanen H, Miettinen A, Laranne J. Utilization of three-dimensional computer-aided preoperative virtual planning and manufacturing in maxillary and mandibular reconstruction with a microvascular fibula flap. J Reconstr Microsurg 2016;32:137-41.

[9] Moro A, Cannas R, Boniello R, Gasparini G, Pelo S. Techniques on modeling the vascularized free fibula flap in mandibular reconstruction. J Craniofac Surg 2009;20:1571-3.

[10] Avraham T, Franco P, Brecht LE, Ceradini DJ, Saadeh PB, Hirsch DL, et al. Functional outcomes of virtually planned free fibula flap reconstruction of the mandible. Plast Reconstr Surg 2014;134:628-34.

[11] van Baar GJC, Forouzanfar T, Liberton NPTJ, Winters HAH, Leusink FKJ. Accuracy of computer-assisted surgery in mandibular reconstruction: a systematic review. Oral Oncol 2018;84:52-60.

[12] Roser SM, Ramachandra S, Blair H, Grist W, Carlson GW, Christensen AM, et al. The accuracy of virtual surgical planning in free fibula mandibular reconstruction: comparison of planned and final results. J Oral Maxillofac Surg 2010;68:2824-32

[13] Naros A, Weise H, Tilsen F, Hoefert S, Naros G, Krimmel M, et al. Three-dimensional accuracy of mandibular reconstruction by patient-specific pre-bent reconstruction plates using an "in-house" 3D-printer. J Craniomaxillofacial Surg 2018;46:1645-51.

[14] Kraeima J, Glas HH, Witjes MJH, Schepman KP. Patient-specific pre-contouring of osteosynthesis plates for mandibular reconstruction: using a three-dimensional key printed solution. J Craniomaxillofac Surg 2018;46:1037-40.

[15] Katakura A, Shibahara T, Noma H, Yoshinari M. Material analysis of ao plate fracture cases. J Oral Maxillofac Surg 2004:62:348-52.

[16] Martola M, Lindqvist C, Hänninen H, Al-Sukhun J. Fracture of titanium plates used for mandibular reconstruction following ablative tumor surgery. J Biomed Mater Res B Appl Biomater 2007;80:345-52.

[17] Sieira Gil R, Roig AM, Obispo CA, Morla A, Pagès CM, Perez JL. Surgical planning and microvascular reconstruction of the mandible with a fibular flap using computer-aided design, rapid prototype modelling, and precontoured titanium reconstruction plates: a prospective study. Br J Oral Maxillofac Surg 2015;53:49-53.

[18] Mottini M, Seyed Jafari SM, Shafighi M, Schaller B. New approach for virtual surgical planning and mandibular reconstruction using a fibula free flap. Oral Oncol 2016;59:6-9.

[19] Sun Y, Luebbers HT, Agbaje JO, Schepers S, Vrielinck L, Lambrichts I, et al. Validation of anatomical landmarks-based registration for image-guided surgery: an in-vitro study. J Craniomaxillofacial Surg 2013;41:522-6.

[20] Wijbenga JG, Schepers RH, Werker PMN, Witjes MJ, Dijkstra PU. A systematic review of functional outcome and quality of life following reconstruction of maxillofacial defects using vascularized free fibula flaps and dental rehabilitation reveals poor data quality. J Plast Reconstr Aesthetic Surg 2016;69:1024-36.

[21] Anne-Gaëlle B, Samuel S, Julie B, Renaud L, Pierre B. Dental implant placement after mandibular reconstruction by microvascular free fibula flap: current knowledge and remaining questions. Oral Oncol 2011;47:1099-104.

[22] Attia S, Wiltfang J, Streckbein P, Wilbrand JF, El Khassawna T, Mausbach K, et al. Functional and aesthetic treatment outcomes after immediate jaw reconstruction using a fibula flap and dental implants. J Craniomaxillofac Surg 2019;47:786-91.

[23] Succo G, Berrone M, Battiston B, Tos P, Goia F, Appendino P, et al. Step-by-step surgical technique for mandibular reconstruction with fibular free flap: application of digital technology in virtual surgical planning. Eur Arch Otorhinolaryngol 2015;272:1491-501.

[24] Yla-Kotola TM, Bartlett E, Goldstein DP, Armstrong K, Gilbert RW, Hofer SO. Union and bone resorption of free fibular flaps in mandibular reconstruction. J Reconstr Microsurg 2013;29:427-32. 